\title{
Congelación de Semen Epididimal Canino con Yema de Huevo Centrifugada
}

\author{
Freezing of Epididymal Canine Sperm With Centrifuged Egg Yolk \\ Giovanni Restrepo B. ${ }^{1,4}$, Carlos Andrés Madrid R. ${ }^{2}$, Laura Prieto R. ${ }^{2}$, \\ Juan Esteban Duque C. ${ }^{3}$, Alexandra Usuga S. ${ }^{2}$
}

\section{Resumen}

El objetivo de esta investigación fue evaluar el uso de yema de huevo centrifugada (YHC) en la congelación de espermatozoides epididimales caninos. Se obtuvieron los complejos testículo-epidídimo (CTE) después de la orquiectomía de 20 perros domésticos (Canis familiaris) de varias razas y en un rango de edad entre 1 y 6 años. Se diseccionó la cola del epidídimo y se obtuvieron los espermatozoides mediante la técnica de flujo retrógrado. Los espermatozoides obtenidos se dividieron en dos alícuotas y se diluyeron en Triladyl ${ }^{\circledR}$ suplementado con YHC al 10\% (YHC10) o YHC al 20\% (YHC20). Se realizó la congelación del semen mediante un protocolo convencional con vapores de nitrógeno líquido en pajillas para $0.5 \mathrm{ml}$. Se utilizó un sistema computarizado para evaluar la movilidad, la cinética y la concentración de los espermatozoides. Adicionalmente, se evaluaron posdescongelación la vitalidad espermática (VE), la morfología anormal (MA) y la integridad de la membrana plasmática (HOS). El análisis estadístico se realizó mediante el ajuste de modelos lineales generalizados (GLM) y la comparación de medias por la prueba de Tukey. Para YHC10 se encontraron valores superiores para las velocidades lineal (VSL), curvilínea (VCL) y media (VAP) en comparación con YHC20 (p<0.05). No se encontraron diferencias relacionadas con la concentración de YHC para otros parámetros de movilidad y cinética espermática, así como para VE, MA y HOS. Se concluye que la YHC puede ser utilizada como suplemento para la congelación de semen epididimal canino. Adicionalmente, cuando la YHC se utiliza en una proporción del 10\% del diluyente, favorece la cinética espermática posdescongelación.

Palabras clave: criopreservación; diluyente; espermatozoide; epidídimo

\footnotetext{
${ }^{1}$ Facultad de Ciencias Agrarias, Universidad Nacional de Colombia, Medellín, Colombia

${ }^{2}$ Facultad de Medicina Veterinaria y Zootecnia, Universidad CES, Medellín, Colombia

${ }^{3}$ Facultad de Ciencias Agrarias, Politécnico Colombiano Jaime Isaza Cadavid, Medellín, Colombia

${ }^{4}$ E-mail: grestre0@unal.edu.co
}

Recibido: 4 de abril de 2017

Aceptado para publicación: 25 de julio de 2017 
The aim of this research was to evaluate the use of centrifuged egg yolk (CEY) in the freezing process of canine epididymal spermatozoa. Testicular-epididymis complexes (TECs) were obtained after the orchiectomy of 20 domestic dogs (Canis familiaris) of different breeds and with an age range between 1 and 6 years. The tail of the epididymis was dissected and spermatozoa were obtained by the retrograde flow technique. The spermatozoa were divided into two aliquots and extended in Triladyl ${ }^{\circledR}$ supplemented with $10 \%$ CEY (CEY10) or 20\% CEY (CEY20). Semen freezing was performed using a standard protocol with liquid nitrogen vapors and semen was packed in straws of $0.5 \mathrm{ml}$. A computerized system was used to evaluate the motility, kinetics and concentration of spermatozoa. In addition, post-thawing sperm vitality (VE), abnormal morphology (MA) and plasma membrane integrity (HOS) were evaluated. Statistical analysis was performed by adjusting generalized linear models (GLM) and comparing means by the Tukey test. For CEY10 higher values for linear (VSL), curvilinear (VCL) and mean (VAP) velocities were found compared to CEY20 $(p<0.05)$. There were no differences related to the concentration of CEY for other parameters as motility, sperm kinetics, VE, MA and HOS. It is concluded that CEY can be used as supplement for the freezing process of canine epididymal semen. Additionally, when the CEY is used in a proportion of $10 \%$ of the extender, it favors the post-thawing sperm kinetics.

Key words: cryopreservation; extender; spermatozoa; epididymis

\section{INTRODUCCIÓN}

La obtención de espermatozoides directamente desde la cola del epidídimo es una técnica para la reproducción asistida, útil como recurso importante en casos de animales de alto valor genético o alta estima, que deben ser esterilizados o que mueren (Mota y Machado, 2012). En los caninos son comunes situaciones como accidentes, intoxicaciones o enfermedades, que súbitamente pueden desencadenar en la orquiectomía, eutanasia o muerte; de manera que la obtención de espermatozoides almacenados en la cola del epidídimo puede ser la única opción para preservar el material genético (Armas et al., 2011). Se han realizado estudios en varias especies animales que han permitido demostrar la factibilidad de recuperar y conservar espermatozoides provenientes del epidídimo, así como desarrollar diferentes métodos de extracción y criopreservación de dichas células (Alvarez y Arismendi, 2006; Monteiro et al., 2009; Jiménez et al., 2013; RibeiroPeres et al., 2014).
Yu y Leibo (2002) observaron que no se produce una reducción significativa de la integridad de la membrana y el acrosoma de espermatozoides caninos recuperados desde los epidídimos, luego de ser refrigerados a $4{ }^{\circ} \mathrm{C}$ durante $48 \mathrm{~h}$, ni tampoco encontraron una disminución evidente de la integridad de la membrana en espermatozoides recuperados después de dos días de tener conservados los epidídimos a $4^{\circ} \mathrm{C}$. En forma coincidente, Armas et al. (2011) indican $48 \mathrm{~h}$ como máximo para recuperar y criopreservar espermatozoides caninos obtenidos de la cola del epidídimo posterior a la orquiectomía. Por otro lado, González et al. (2013) observaron una reducción progresiva de la movilidad, la integridad de membrana y la integridad del acrosoma entre las 3 y las $72 \mathrm{~h}$ de almacenamiento post mortem y posdescongelación. Otros investigadores han evaluado aspectos como el uso de crioprotectores, las tasas de congelación y los métodos de separación espermática para la criopreservación de semen epididimal canino (Hewitt et al., 2001; Hishinuma y Sekine, 2004; Ponglowhapan y Chatdarong, 2008). 
Otro punto de interés ha sido el uso de diluyentes o suplementos apropiados para la criopreservación de espermatozoides epididimales caninos. Tittarelli et al. (2006) compararon la conservación de la calidad del semen de epidídimos almacenado a $4{ }^{\circ} \mathrm{C}$ por 24, 48 o $72 \mathrm{~h}$, diluido en solución salina isotónica estéril o en diluyente tris-yema de huevo $(\mathrm{YH})$, hallando solo a favor de este último, diferencias en la integridad del acrosoma. Nöthling et al. (2007) encontraron que el diluyente Biladyl suplementado con pasta Equex STM es más adecuado para la congelación de los espermatozoides epididimales caninos que el diluyente Andromed, y que el líquido prostático mejora la congelabilidad y la longevidad posdescongelación de dichas células. Mota et al. (2014) utilizaron los diluyentes ACP$106 \mathrm{c}$ (diluyente de agua de coco en polvo) y TRIS para la congelación espermática, con resultados equivalentes posdescongelación para ambos diluyentes en cuanto a la movilidad, la cinética, la morfología y la integridad de la membrana de los espermatozoides.

En diferentes reportes de criopreservación de semen canino, la YH ha sido un aditivo común en los diluyentes, en concentraciones que varían entre el 5, 10 y $20 \%$ (Barbosa et al., 2009; Martins et al., 2012; Cardoso et al., 2010). El efecto protector de la YH ha sido atribuido principalmente a su fracción de lipoproteínas de baja densidad (LDL), responsable de la resistencia al choque térmico y la mejora de la movilidad, a través de la prevención de la pérdida de los fosfolípidos de la membrana (Amirat et al., 2004; Cardoso et al., 2010). Sin embargo, se conoce que la YH genera riesgo microbiológico y alteraciones de la movilidad, la integridad del acrosoma y la fosforilación oxidativa de los espermatozoides; además, ocasiona complicaciones para realizar investigaciones bioquímicas, metabólicas y microscópicas del semen (Wall y Foote, 1999; Moussa et al., 2002; Sariozkan et al., 2010). De otro lado, el uso de altas proporciones de $\mathrm{YH}$ requiere la adición de detergentes para la solubilización de la misma, como es el caso de la pasta
Equex STM (Nöthling et al., 2007; AbdelMalak et al., 2015).

Un estudio reciente demostró que la suplementación del diluyente con LDL mejora la calidad de los espermatozoides epididimales caninos durante la congelación, especialmente con un efecto favorable sobre la integridad de la membrana plasmática (Prapaiwan et al., 2016). Sin embargo, la obtención de LDL puede ser limitada por su alto costo o por procesos de extracción que, aunque son eficientes, podrían considerarse dispendiosos y prolongados, además de requerir aditivos y equipos de centrifugación de alto rendimiento (10 000-50 $000 \mathrm{~g}$ ) (Wall y Foote, 1999; Moussa et al., 2002; Pillet et al., 2011). Una alternativa está en el uso de yema de huevo centrifugada (YHC), la cual posee una menor cantidad de constituyentes de $\mathrm{YH}$ y proporciona una mayor protección de la movilidad, la integridad de membrana y la morfología de los espermatozoides en los procesos de congelación-descongelación (Nouri et al., 2013). Adicionalmente, la producción de YHC puede realizarse mediante procesos más simples y es adecuada para realizar estudios espermáticos por sistemas computarizados (CASA), al permitir la reducción de la viscosidad del diluyente (Nouri et al., 2013; Restrepo et al., 2016).

El objetivo de esta investigación fue evaluar el uso de YHC en la congelación de espermatozoides epididimales caninos.

\section{Materiales y Métodos}

\section{Obtención de Muestras Biológicas}

Se obtuvieron 40 complejos testículoepidídimo (CTE) después de la orquiectomía de 20 perros domésticos (Canis familiaris) de varias razas, con edades entre 1 y 6 años, ubicados y mantenidos en iguales condiciones de ambiente, manejo y alimentación, en un criadero del municipio de Bello (Antioquia, Colombia). Los CTE se transportaron y mantuvieron en refrigeración a $5{ }^{\circ} \mathrm{C}$ durante $2 \mathrm{~h}$. 
Una vez en el laboratorio se lavaron con solución salina fisiológica ( $\mathrm{NaCl}$ al $0.9 \%$ ), se procedió con la separación de los epidídimos de los CTE y se removió por disección el tejido conjuntivo. Se diseccionaron la cola del epidídimo y el conducto deferente proximal de acuerdo a lo descrito por Hewitt et al. (2001). Los espermatozoides de la cauda epididimal se recuperaron mediante la técnica de flujo retrógrado previamente reportada en caninos por Mota et al. (2014), para lo cual, se utilizaron $20 \mathrm{ml}$ del diluyente EquiPlus $^{\circledR}$ (Minitube, Alemania). Las muestras provenientes de cada par de epidídimos se mezclaron después de su extracción.

\section{Procesamiento y Evaluación Espermática}

Después de la recuperación epididimal se realizó la evaluación de la movilidad, cinética y concentración de los espermatozoides por animal, mediante un análisis computarizado con el sistema Sperm Class Analizer (SCA ${ }^{\circledR}$, Microptic, España) de acuerdo a un protocolo modificado del reportado por Restrepo et al. (2013), en cuanto a la configuración de especie perro y área de la partícula entre 12 y $80 \mathrm{um}^{2}$. Se utilizó un microscopio de contraste de fase (Eclipse E200, Nikon, Japón) con una cámara digital (Scout SCA780, Basler, EEUU). Se determinaron los parámetros de movilidad total (MT), movilidad progresiva (MP), velocidad lineal (VSL), velocidad curvilínea (VCL), velocidad media (VAP), índice de linealidad (LIN), índice de rectitud (STR), índice de oscilación (IO), amplitud lateral de la cabeza (ALH), frecuencia de batida (BCF) y recorridos circulares (RC).

La suspensión de espermatozoides recuperada se separó en dos alícuotas de $10 \mathrm{ml}$ y cada una se sometió a un proceso de separación espermática, para lo cual se depositó en el fondo del tubo $1 \mathrm{ml}$ de CushionFluid ${ }^{\mathrm{TM}}$ (Minitube, Alemania) y luego se centrifugó a $800 \mathrm{~g}$ durante $15 \mathrm{~min}$ (Mikro 220R, Hettich, Alemania). El precipitado se resuspendió en un diluyente para congelación de semen (Triladyl, Minitube, Alemania) suplementado con YHC al 10\% (YHC10) o YHC al 20\% (YHC20). La YHC se obtuvo de acuerdo a un protocolo modificado del descrito por Nouri et al. (2013). Los huevos frescos de gallina fueron rotos manualmente, las yemas se separaron de la albúmina y se cubrieron con un papel de filtro para eliminar chalazas y trazas de albúmina adheridas a la membrana vitelina. Con una jeringa se aspiró la yema y se diluyó en proporción 3:1 en agua ultra pura. Luego se centrifugó a $1600 \mathrm{~g}$ durante 99 min a temperatura ambiente. Después de la centrifugación, se eliminó el material lipídico en la parte superior de cada tubo, se recuperó la fracción transparente soluble en agua y se descartó el sedimento en la parte inferior del tubo.

\section{Congelación de Semen Epididimal}

La congelación del semen se realizó mediante un protocolo de congelación convencional, para lo cual el semen se sometió a refrigeración durante $30 \mathrm{~min}$ a $5{ }^{\circ} \mathrm{C}$ y luego se empacó en pajillas para $0.5 \mathrm{ml}$, a una concentración de $100 \times 10^{6}$ células $/ \mathrm{ml}$, mediante una máquina de empaque y sellado automático (MRS1 Dual V2, IMV Technologies, Francia). Las pajillas se ubicaron de forma horizontal a $4 \mathrm{~cm}$ de distancia de la superficie del nitrógeno líquido durante 10 min y luego se sumergieron en el tanque (Rota et al., 2006). Después de un mes de almacenamiento, las pajillas se descongelaron en agua a $37^{\circ} \mathrm{C}$ por 1 min y se procedió a la evaluación posdescongelación.

\section{Evaluación Seminal Posdescongelación}

Se realizó la evaluación posdescongelación de cuatro pajillas de semen por animal $(n=80)$. Se evaluaron los parámetros de movilidad y cinética espermática como se describió previamente. De forma adicional, se realizó la evaluación de la morfología y la vitalidad de los espermatozoides mediante la tinción con eosina-nigrosina (Barth y Oko, 1989). Sobre una platina a $37^{\circ} \mathrm{C}$ se mezcló una gota de semen con una gota del colorante, se realizó un extendido y se determinó a 
través de microscopía de contraste de fase (Eclipse E200, Nikon, Japón) la proporción de células con morfología anormal (MA) y membrana estructuralmente íntegra o vitalidad espermática (VE). Para cada prueba se evaluaron 200 espermatozoides.

Además, se evaluó la integridad funcional de la membrana plasmática mediante la prueba hipoosmótica (HOS) de acuerdo a lo descrito por Pinto y Kozink (2008). Se tomaron $100 \mu 1$ de semen y se adicionaron a un tubo con $500 \mu 1$ de una solución hipoosmótica de sacarosa 5.4\% (100 mOsmol/1). Esta mezcla se incubó a $38.5^{\circ} \mathrm{C}$ por $30 \mathrm{~min}$ y luego se evaluó la reacción de 200 espermatozoides por microscopía de contraste de fase (Eclipse E200, Nikon, Japón).

\section{Análisis Estadístico}

Los resultados de calidad seminal se analizaron mediante el ajuste de modelos lineales generalizados (GLM) para cada variable dependiente. En cada modelo se incluyeron los efectos fijos del canino y de la concentración de YHC. Se evaluó la normalidad de los datos mediante la prueba de ShapiroWilk. La comparación de medias se realizó mediante la prueba de Tukey. Todas las evaluaciones se realizaron con el programa SAS 9.2. (SAS Inst., EEUU).

\section{Resultados y Discusión}

La criopreservación de espermatozoides epididimales tiene como objetivo mantener la disponibilidad de germoplasma masculino para su uso futuro (Prapaiwan et al., 2016). Sin embargo, diversos factores como el método de extracción desde el epidídimo, el tiempo entre la recuperación y la congelación, el diluyente utilizado y el uso de fluido prostático, influyen drásticamente en la calidad de los espermatozoides epididimales caninos congelados (Martins et al., 2012; Korochkina et al., 2014; Mota et al., 2014; Hori et al., 2015). Entre los suplementos co- múnmente utilizados en los diluyentes de congelación está la YH, conocida por su capacidad crioprotectora (Ponglowhapan et al., 2006; Nöthling et al., 2007). En esta investigación, se evaluó el uso de YHC como alternativa de suplementación, dadas las limitantes generadas por el uso de YH (Wall y Foote, 1999; Moussa et al., 2002; Sariozkan et al., 2010).

Se encontraron $547.5 \pm 448.9 \times 10^{6}$ espermatozoides por animal (ambos epidídimos). Hori et al. (2015) encontraron un número similar de espermatozoides recuperados desde epidídimos $\left(595.8 \pm 90.2 \times 10^{6}\right)$; sin embargo, la alta variabilidad observada en el presente estudio pudo ser debida a factores como la raza y la edad, toda vez que en el estudio citado se trabajó con perros Beagle y un rango de edad más estrecho (1.0 a 3.5 años). Hewitt et al. (2001), encontraron igualmente una alta variabilidad en la concentración de espermatozoides epididimales (4.6$400 \times 10^{6} / \mathrm{ml}$ ) en perros de varias razas y en un rango de 0.5 a 6 años.

Los resultados para los parámetros de movilidad y cinética espermática del semen epididimal recuperado se presentan en el Cuadro 1. Los resultados fueron similares a los reportados por Mota et al. (2014), a excepción de valores mayores para VCL (88.8 $\pm 4.3 \mu \mathrm{m} / \mathrm{seg}), \operatorname{VSL}(70.2 \pm 2.5 \mu \mathrm{m} / \mathrm{seg}) \mathrm{y}$ $\operatorname{VAP}(78.7 \pm 2.4 \mu \mathrm{m} / \mathrm{seg})$, así como para los índices calculados a partir de dichos valores, LIN $(62.5 \pm 3.0 \%)$ y STR $(80.3 \pm 1.4 \%)$. De otro lado, Korochkina et al. (2014) hallaron resultados similares al presente trabajo para VCL, VSL y VAP, pero un valor de MT inferior al encontrado en el mismo $(29.2 \pm 20.0 \%$ vs. $53.6 \pm 14.7 \%$ ).

Se conoce que el tiempo transcurrido entre la obtención de los epidídimos y la extracción de los espermatozoides, así como el tiempo de almacenamiento de los mismos, son determinantes en la movilidad espermática (Yu y Leibo, 2002). En esta investigación, el transporte de los epidídimos así como la extracción y el procesamiento de los esper- 
Cuadro 1. Movilidad y cinética de espermatozoides obtenidos de epidídimos caninos posorquiectomía $(\mathrm{n}=20)$

\begin{tabular}{lcccccc}
\hline Variable & Media & DE & CV & EE & Min & Max \\
\hline MT $(\%)$ & 53.6 & 14.7 & 27.4 & 3.2 & 21.9 & 79.4 \\
MP $(\%)$ & 23.6 & 11.9 & 50.6 & 2.6 & 6.5 & 52.5 \\
VCL $(\mu \mathrm{m} / \mathrm{seg})$ & 73.4 & 14.5 & 19.7 & 3.1 & 49.6 & 105.2 \\
VSL $(\mu \mathrm{m} / \mathrm{seg})$ & 35.2 & 10.2 & 29.0 & 2.2 & 17.3 & 53.8 \\
VAP $(\mu \mathrm{m} / \mathrm{seg})$ & 44.2 & 10.3 & 23.4 & 2.2 & 29.0 & 67.0 \\
LIN $(\%)$ & 47.7 & 9.6 & 20.1 & 2.1 & 30.9 & 72.2 \\
STR $(\%)$ & 78.7 & 7.7 & 9.8 & 1.6 & 56.6 & 90.2 \\
IO $(\%)$ & 60.1 & 7.1 & 11.9 & 1.5 & 47.7 & 80.0 \\
ALH $(\mu \mathrm{m})$ & 4.3 & 0.6 & 13.7 & 0.1 & 3.0 & 5.3 \\
BCF $(\mathrm{Hz})$ & 9.3 & 1.5 & 16.4 & 0.3 & 5.9 & 13.0 \\
RC $(\%)$ & 30.5 & 15.6 & 51.4 & 3.4 & 0 & 57.0 \\
\hline
\end{tabular}

DE: desviación estándar; CV: coeficiente de variación; EE: error estándar; Min: valor mínimo; Max: valor máximo

MT: movilidad total; MP: movilidad progresiva; VSL: velocidad lineal; VCL: velocidad curvilínea; VAP: velocidad media; LIN: índice de linealidad; STR: índice de rectitud; IO: índice de oscilación; ALH: amplitud lateral de la cabeza; BCF: frecuencia de batida; RC: recorridos circulares

Cuadro 2. Movilidad y cinética posdescongelación de semen epididimal canino congelado con yema de huevo centrifugada (YHC) $(\mathrm{n}=80$ pajillas)

\begin{tabular}{lcc}
\hline Variable & YHC10 & YHC20 \\
& Media \pm DE & Media \pm DE \\
\hline MT $(\%)$ & $27.4 \pm 9.3^{\mathrm{a}}$ & $27.9 \pm 10.5^{\mathrm{a}}$ \\
MP $(\%)$ & $12.9 \pm 5.7^{\mathrm{a}}$ & $11.7 \pm 5.2^{\mathrm{a}}$ \\
VCL $(\mu \mathrm{m} / \mathrm{seg})$ & $79.9 \pm 13.7^{\mathrm{a}}$ & $72.4 \pm 9.9^{\mathrm{b}}$ \\
VSL $(\mu \mathrm{m} / \mathrm{seg})$ & $57.9 \pm 15.0^{\mathrm{a}}$ & $51.4 \pm 11.0^{\mathrm{b}}$ \\
VAP $(\mu \mathrm{m} / \mathrm{seg})$ & $63.8 \pm 14.0^{\mathrm{a}}$ & $57.1 \pm 9.9^{\mathrm{b}}$ \\
LIN $(\%)$ & $71.6 \pm 9.5^{\mathrm{a}}$ & $70.7 \pm 9.4^{\mathrm{a}}$ \\
STR $(\%)$ & $89.8 \pm 4.9^{\mathrm{a}}$ & $89.4 \pm 4.9^{\mathrm{a}}$ \\
IO $(\%)$ & $79.4 \pm 6.8^{\mathrm{a}}$ & $78.7 \pm 6.7^{\mathrm{a}}$ \\
ALH $(\mu \mathrm{m})$ & $3.1 \pm 0.7^{\mathrm{a}}$ & $3.0 \pm 0.7^{\mathrm{a}}$ \\
BCF $(\mathrm{Hz})$ & $11.1 \pm 1.5^{\mathrm{a}}$ & $11.0 \pm 2.1^{\mathrm{a}}$ \\
RC $(\%)$ & $7.9 \pm 5.1^{\mathrm{a}}$ & $8.9 \pm 6.6^{\mathrm{a}}$ \\
\hline
\end{tabular}

$a, b$ Letras diferentes dentro de filas indican diferencia estadística $(p<0.05)$

DE: desviación estándar; YHC10: yema de huevo centrifugada al 10\%; YHC20: yema de huevo centrifugada al $20 \%$

MT: movilidad total; MP: movilidad progresiva; VSL: velocidad lineal; VCL: velocidad curvilínea; VAP: velocidad media; LIN: índice de linealidad; STR: índice de rectitud; IO: índice de oscilación; ALH: amplitud lateral de la cabeza; BCF: frecuencia de batida; RC: recorridos circulares 
matozoides se realizaron antes de los periodos relacionados en la literatura con la disminución de la viabilidad de estas células epididimales (Armas et al., 2011; González et al., 2013). Otro factor que pudiese afectar la movilidad de los espermatozoides epididimales caninos es el diluyente utilizado para su recuperación. Nöthling et al. (2007) observaron marcadas diferencias en la movilidad progresiva a favor de un diluyente con YH (Biladyl) en comparación de un diluyente sin dicho suplemento (Andromed). En esta investigación, el diluyente utilizado para la extracción de los espermatozoides desde los epidídimos no contenía YH.

Los resultados para la movilidad y la cinética posdescongelación de los espermatozoides epididimales, congelados con dos concentraciones de YHC se presentan en el Cuadro 2. No se observaron diferencias en la movilidad total y progresiva del semen descongelado, ni en la mayoría de los parámetros cinéticos; sin embargo, se encontraron resultados superiores para VCL, VSL y VAP de los espermatozoides epididimales congelados con YHC10 en comparación con el semen congelado con YHC20 $(\mathrm{p}<0.05)$. Lo anterior puede ser relevante, toda vez que se conoce que existe una relación favorable entre el incremento de VCL, VSL y VAP con la capacidad fertilizante del semen canino (Silva et al., 2006).

Los valores para MT y MP fueron superiores a los reportados por Hewitt et al. (2001), cercanos a los hallados por Hori et al. (2015) e inferiores a los reportados por Martins et al. (2012) y Abdel-Malak et al. (2015). Sin embargo, en estas dos últimas investigaciones solo se usaron muestras de semen epididimal con una movilidad mínima de 80 y $75 \%$, respectivamente, mientras que en la presente investigación no se establecieron criterios de exclusión de las muestras procesadas. De otro lado, Nöthling et al. (2007) encontraron bajo condiciones similares a la presente investigación, un valor promedio de MP $(12.6 \pm 0.7 \%)$ muy cercano a los aquí reportados (Cuadro 2).
No se hallaron reportes previos del uso de YHC en la congelación de semen epididimal canino; sin embargo, en un estudio de Mota et al. (2014) se evaluó la congelación de semen epididimal canino con diluyentes suplementados con $10 \%$ de $\mathrm{YH}$ (ACP-106c) y con $20 \%$ de YH (TRIS), encontrando resultados posdescongelación superiores de VSL y STR para el semen criopreservado con $10 \%$ de YH. Ambos trabajos muestran un efecto de la concentración de YH o YHC en la cinética espermática, que podría ser explicada por factores como la viscosidad del diluyente o el aporte de LDL (Moussa et al., 2002; Nouri et al., 2013). Otros estudios reportan que el uso de LDL provenientes de $\mathrm{YH}$ en la congelación de semen canino (eyaculado), mejora de forma ostensible la movilidad, así como la integridad del ADN, de la membrana plasmática y del acrosoma, en comparación con la YH (Bencharif et al., 2008; 2010). Así mismo el uso de LDL mejora la integridad de la membrana plasmática de espermatozoides epididimales caninos congelados (Prapaiwan et al., 2016).

No se observaron diferencias significativas debidas a la proporción de YHC sobre la morfología, la vitalidad o la integridad de la membrana plasmática de los espermatozoides epididimales congelados-descongelados (Figura 1). Mota et al. (2014) tampoco hallaron diferencias posdescongelación en la integridad de membrana (HOS) utilizando diluyentes con 10 y $20 \%$ de $\mathrm{YH}$; sin embargo, estos autores encontraron una mayor proporción de espermatozoides morfológicamente normales en el diluyente con $10 \%$ de $\mathrm{YH}$.

En el presente trabajo se observó una menor proporción de espermatozoides anormales en comparación con otras investigaciones donde se realizó la congelación de semen epididimal canino (Nöthling et al., 2007; Martins et al., 2012). Así mismo, se han reportado resultados similares de vitalidad espermática (Hori et al., 2015) e integridad de la membrana plasmática (Prapaiwan et $a l ., 2016)$ en semen epididimal canino criopreservado. 


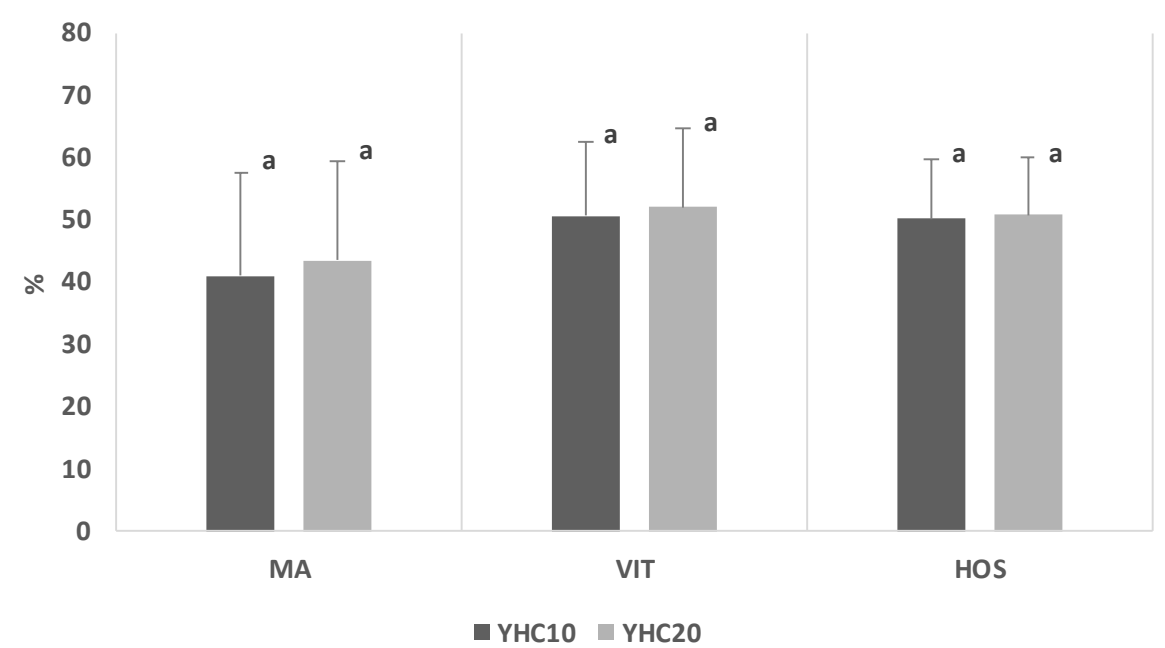

Figura 1. Morfología, vitalidad e integridad de membrana posdescongelación de semen epididimal canino congelado con yema de huevo centrifugada (YHC).

Barras superiores indican la desviación estándar. Letras iguales indican ausencia de diferencias estadísticas.

YHC10: yema de huevo centrifugada al 10\%. YHC20: yema de huevo centrifugada al 20\%. MA: morfología anormal. VIT: vitalidad espermática. HOS: integridad funcional de la membrana plasmática

\section{Conclusiones}

- La yema de huevo centrifugada (YHC) puede ser utilizada como suplemento en el diluyente para la congelación de semen epididimal canino.

- El uso de un 10\% de YHC en el diluyente de congelación, favorece la cinética posdescongelación de los espermatozoides epididimales, en comparación al uso de un $20 \%$ de este suplemento.

\section{Literatura Citada}

1. Abdel-Malak M, Mahmoud A, Hussein F, Metwally K. 2015. Effect of adding Equex STM paste and BHT to different extenders on viability, plasma membrane and DNA integrity of German Shepherd dog spermatozoa during freezing and thawing methods in Egypt. Assiut Vet Med J 61: 242-256.
2. Alvarez M, Arismendi D. 2006. Movilidad individual de los espermatozoides epididimarios de toros post mortem obtenidos mediante lavado retrógrado. Zootecnia Trop 24: 267-280.

3. Amirat L, Tainturier D, Jeanneau L, Thorin C, Gérard O, Courtens J, Anton M. 2004. Bull semen in vitro fertility after cryopreservation using egg yolk LDL: a comparison with Optidyl, a commercial egg yolk extender. Theriogenology 61: 895-907. doi: 10.1016/S0093-691X(03)00259-0

4. Armas S, Fernández A, Vásquez M, Santiani A. 2011. Determinación del tiempo máximo para recuperar y criopreservar espermatozoides obtenidos de la cola del epidídimo en caninos post orquiectomía. Rev Inv Vet Perú 22: 199205. doi: 10.15381/rivep.v22i3.257

5. Barbosa C, Madeira V, Jucá R, Oliveira A, Uchoa D, Silva L. 2009. Criopreservação de sêmen canino com um diluidor à base de água de coco na 
forma de pó (ACP-106): efeito da temperatura de adição do glicerol (27 e 4 $\left.{ }^{\circ} \mathrm{C}\right)$. Ciência Anim Bras 10: 1209-1217.

6. Barth A, Oko, R. 1989 Abnormal morphology of bovine spermatozoa. Iowa, USA: Iowa State University Press. 285 p.

7. Bencharif D, Amirat L, Anton M, Schmitt E, Desherces S, Delhomme G, Langlois M, et al. 2008. The advantages of LDL (low density lipoproteins) in the cryopreservation of canine semen. Theriogenology 70: 14781488. doi: $10.1016 / \mathrm{j}$.theriogenology.2008.06.095

8. Bencharif D, Amirat L, Pascal O, Anton M, Schmitt E, Desherces S, Delhomme G, et al. 2010. The advantages of combining low-density lipoproteins with glutamine for cryopreservation of canine semen. Reprod Domest Anim 45: 189-200. doi: 10.1111/j.1439-0531.2008.01198.x

9. Cardoso J, Paula N, Uchoa D, Silva L. 2010. Diferentes concentrações de gema de ovo na qualidade do sêmen canino diluído em $\mathrm{ACP}^{\circledR}$-106 e resfriado a $4{ }^{\circ} \mathrm{C}$. Comunicata Sci 1: 146-152.

10. González J, Tadeo J, Ortega C, Toledano A, Vergara M, Ávalos A. 2013. Criopreservación de espermatozoides epididimales a diferentes tiempos postmortem en caninos. Rev Salud Anim 35: 137-141.

11. Hewitt D, Leahy $R$, Sheldon I, England G 2001. Cryopreservation of epididymal dog sperm. Anim Reprod Sci 67: 101-111. doi: 10.1016/S03784320(01)00090-2

12. Hishinuma M, Sekine J. 2004. Separation of canine epididymal spermatozoa by Percoll gradient centrifugation. Theriogenology 61: 365-372. doi: 10.1016/S0093-691X(03)00219-X

13. Hori T, Atago T, Kobayashi M, Kawakami E. 2015. Influence of different methods of collection from the canine epididymides on post-thaw caudal epididymal sperm quality. J Vet Med Sci 77: 625-630. doi: 10.1292/jvms. 14-0421
14. Jiménez E, Pérez-Marín C, Vizuete G, Millán Y, Agüera E. 2013. Effect of different extenders on in vitro characteristics of feline epididymal sperm during cryopreservation. Reprod Domest Anim 48: 665-672. doi: 10.1111/rda. 12142

15. Korochkina E, Johannisson A, Goodla L, Morrell J, Axner E. 2014. Effect of prostatic fluid on the quality of fresh and frozen-thawed canine epididymal spermatozoa. Theriogenology 82: 12061211. doi: $0.1016 / j$.theriogeno-logy.2014.06.024

16. Martins M, Justino R, Sant'anna M, Trautwein L, Souza F. 2012. Comparison of two different extenders for cryopreservation of epididymal dog sperm. Reprod Dom Anim 47: 293-294. doi: 10.1111/rda.12042

17. Monteiro G, Guasti P, Papa F. 2009. Colheita e preservação de células espermáticas de garanhões recuperadas da cauda do epididímo. Vet Zootec 16: 448-458.

18. Mota A, Machado L. 2012. Recuperação e conservação de espermatozoides epididimários de mamíferos. Acta Vet Brasilica 6: 1-8. doi: 10.21708/avb.2012.6.1.2880

19. Mota A, Rodrigues H, Pereira T, Barbosa de Souza M, Azevedo de Freitas L, Alencar de Araújo A, Machado da Silva L. 2014. Cryopreservation of canine epididymal sperm using ACP-106c and TRIS. Cryobiology 69: 17-21. doi: 10.1016/j.cryobiol.2014.04.013

20. Moussa M, Martinet V, Trimeche A, Tainturier D, Anton M. 2002. Low density lipoproteins extracted from hen egg yolk by an easy method: cryoprotective effect on frozen-thawed bull semen. Theriogenology 57: 1695-1706. doi: 10.1016/S0093-691X(02)00682-9

21. Nöthling J, Gerber D, Colenbrander B, Dijkstra M, Bakker T, De Cramer K. 2007. The effect of homologous prostatic fluid on motility and morphology of dog epididymal spermatozoa extended 
and frozen in Biladyl with Equex STM paste or Andromed. Theriogenology 67: 264-275. doi: 10.1016/j.theriogenology.2006.07.018

22. Nouri H, Towwhidi A, Zhandi M, Sadeghi R. 2013. The effects of centrifuged egg yolk used with INRA plus soybean lecithin extender on semen quality to freeze Caspian horse semen. J Equi Vet Sci 33: 1050-1053. doi: 10.1016/j.jevs.2013.03.184

23. Pillet E, Duchamp G, Batellier F, Beaumal V, Anton M, Desherces S, Schmitt E, Magistrini M. 2011. Egg yolk plasma can replace egg yolk in stallion freezing extenders. Theriogenology 75: 105-114. doi: 10.1016/ j.theriogenology.2010.07.015

24. Pinto C, Kozink D. 2008. Simplified hypoosmotic swelling testing (HOST) of fresh and frozen-thawed canine spermatozoa. Anim Reprod Sci 104: 450-455. doi: 10.1016/j.anireprosci.2007.07.005

25. Ponglowhapan S, Chatdarong K, Sirivaidyapong S, Lohachit C. 2006. Freezing of epididymal spermatozoa from dogs after cool storage for 2 or 4 days. Theriogenology 66: 1633-1636. doi: 10.1016/j.theriogenology.2006.01.032

26. Ponglowhapan S, Chatdarong $K$. 2008. Effects of Equex STM Paste on the quality of frozen-thawed epididymal dog spermatozoa. Theriogenology 69: 666-672. doi: 10.1016/j.theriogenology.2007.11.005

27. Prapaiwan N, Tharasanit T, Punjachaipornpol S, Yamtang D, Roongsitthichai A, Moonarmart W, Kaeoket K, Manee-in S. 2016. Lowdensity lipoprotein improves motility and plasma membrane integrity of cryopreserved canine epididymal spermatozoa. Asian-Australas J Anim Sci 29: 646-651. doi: 10.5713/ajas.15.0572

28. Restrepo G, Ocampo D, Velásquez A. 2013. Evaluación de la movilidad del semen criopreservado de caballos criollo colombiano por un sistema analizador de clase. Rev UDCA Act Div Cient, 16: 445-450.
29. Restrepo G, Rodríguez L, Duque J. 2016. Proliferación microbiana y calidad posdescongelación de semen equino criopreservado en presencia de antibióticos. Rev Inv Vet Perú 27: 316325. doi: 10.15381/rivep.v27i2.11650

30. Ribeiro-Peres A, Munita Barbosa L, Yumi-Kanazawa M, Mello-Martins M, Ferreira de Souza F. 2014. Criopreservación de espermatozoides bovinos extraídos de la cola del epidídimo utilizando los métodos convencional y automatizado. Arch Med Vet 46: 31-38. doi: 10.4067/S0301-732X2014000100005

31. Rota A, Milani C, Cabianca G, Martini $M$. 2006. Comparison between glycerol and ethylene glycol for dog semen cryopreservation. Theriogenology 65: 1848-1858. doi: 10.1016/j.theriogenology.2005.10.015

32. Sariozkan S, Tuncer P, Bucak M, Buyukleblebici S, Kinet H, Bilgen A. 2010. The effects of different egg yolk concentrations used with soy bean lecithin-based extender on semen quality to freeze bull semen. Eurasian J Vet Sci 26: 45-49.

33. Silva A, Cardoso R, Silva L. 2006. Influence of temperature during glycerol addition and post-thaw dilution on the quality of canine frozen semen. Reprod Domest Anim 41: 74-78. doi: 10.1111/ j.1439-0531.2006.00630.x

34. Tittarelli C, Savignone C, Arnaudín E, Stornelli M.C, Stornellic M.A, Luzbel de la Sota R. 2006. Effect of storage media and storage time on survival of spermatozoa recovered from canine and feline epididymides. Theriogenology 66: 1637-1640. doi: 10.1016/j.theriogenology.2006.01.021

35. Wall R, Foote $R$. 1999. Fertility of bull semen frozen and store in clarified egg yolk-Tris-glycerol extender. J Dairy Sci 82: 817-821. doi: $10.3168 / j d s . S 0022-$ 0302(99)75301-4

36. Yu I, Leibo P. 2002. Recovery of motile, membrane-intact spermatozoa from canine epididymides stored for 8 days at $4^{\circ} \mathrm{C}$. Theriogenology 57: 1179-1190. doi: 10.1016/S0093-691X(01)00711-7 